\title{
First Reported Mycobacterium Marinum Infection Case in a Patient With Psoriatic Arthritis Maintained on Golimumab
}

\author{
Mohamad Ali RIDA ${ }^{1}$ (D) Tara BARDAWIL ${ }^{2}$ (D) Dima IBRAHIM ${ }^{3}$ (D) \\ ${ }^{1}$ Univeristy of Toronto, Rheumatology, Toronto, Canada \\ ${ }^{2}$ Department of Dermatology, American Univerisity of Beirut Medical Center, Beirut, Lebanon \\ ${ }^{3}$ Department of Infectious Diseases, American University of Beirut Medical Center, Beirut, Lebanon
}

Tumor necrosis factor (TNF) inhibition has become a widely used biological therapy in patients with underlying autoimmune diseases and its association with increased risk for tuberculosis and other granulomatous diseases has been extensively studied recently. ${ }^{1}$ Nontuberculous mycobacterial infections are also associated with the immune dysfunction caused by the use of immunomosdulatory drugs, mainly anti TNF-alpha $(\alpha)$, ranging from localized skin infection to disseminated disease. ${ }^{2,3}$ Psoriasis and psoriatic arthritis patients treated with anti-TNF are at higher risk of developing skin non-tuberculous mycobacterial infection due to the disease itself and the combined side effect of the medications used. ${ }^{4}$ The following case describes a skin infection with Mycobacterium marinum (M. marinum) associated with the use of golimumab, a fully human monoclonal antibody against TNF- $\alpha$, in a patient with psoriatic arthritis.

A 53-year-old female patient with psoriatic arthritis followed at the rheumatologydermatology clinic presented for evaluation of a non-resolving indurated plaque over the base of the right fifth digit despite the use of local steroid and systemic treatment for her arthritis. She reported no recent trauma or abrasion, no fever or chills. No other skin lesions were identified back then. Golimumab $50 \mathrm{mg}$ subcutaneous monthly injections were added to standard methotrexate for worsening arthritis and liver function tests secondary to methotrexate. The patient received 2 doses of golimumab $50 \mathrm{mg}$ after which she started noticing the erythematous lesion over her finger, increasing in size and erythema (Figure 1a). Informed consent of the patient was obtained from the patient for this case report.

She was referred to the dermatology for skin biopsy and started empirically on a course of amoxicillin for suspected cellulitis. Golimumab was stopped and the patient was kept on methotrexate to avoid flare of her joint disease.

Pathology was consistent with granulomatous tuberculoid dermatitis (Figure 1b). Acid fast, Gram, periodic acid-Schiff, and Gomori methenaminesilver stains were non-revealing for organisms or fungi. Non-tuberculous mycobacteria were recovered from the mycobacterial culture one month after initial presentation and were identified later as M. marinum.

The patient was then started on clarithromycin and doxycycline with significant improvement after two weeks of treatment.

\footnotetext{
Received: July 09, 2019 Accepted: July 21, 2019 Published online: February 07, 2020

Correspondence: Mohamad Ali Rida, MD. UHN - Toronto Western Hospital, 399 Bathurst St., Toronto, ON M5T 2S8, Canada. Tel: +14377773254 e-mail: mohamad.rida@uhnresearch.ca 


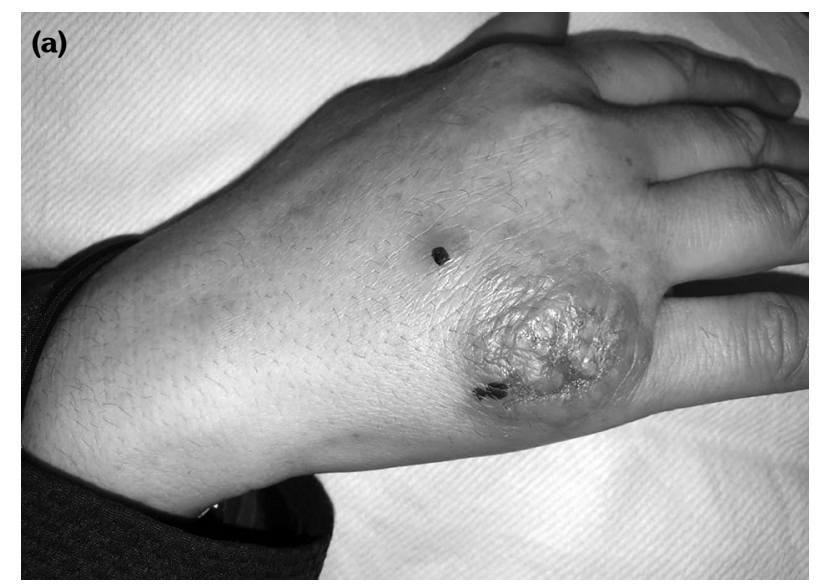

(b)

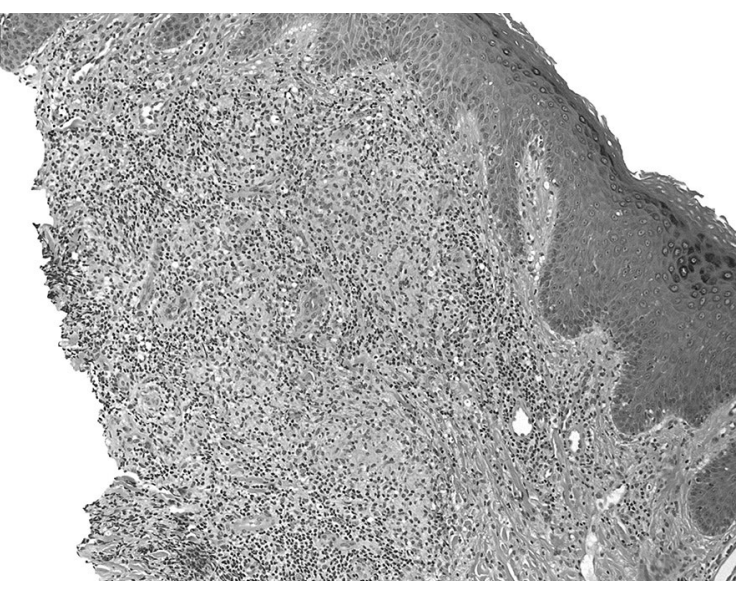

Figure 1. (a) Non-resolving indurated plaque over base of right fifth digit. (b) Biopsy consistent with granulomatous tuberculoid dermatitis $(\mathrm{H}-\mathrm{E} \times 100)$.

Mycobacterium marinum is an atypical mycobacteria or mycobacteria other than Mycobacterium tuberculosis that is typically associated with fishes and water. ${ }^{5}$ Few cases of M. marinum infection were reported in patients treated with anti-TNF- $\alpha$ ranging from localized skin infection to disseminated disease..$^{2,3}$

The literature mentions only six cases of psoriasis associated with $M$. marinum infection and anti-TNF- $\alpha$ was administered in all cases. ${ }^{4,6-9}$ This is another rare case of $M$. marinum sporotrichoid-like skin infection in a patient on anti-TNF- $\alpha$ treatment.

To our best knowledge, the present case is the first described in association with golimumab therapy. The exposure to fresh fish as well the immunosuppression predisposed the patient to acquire this infection. Golimumab was stopped and the patient was started on combination clarithromycin and doxycycline with significant improvement after two weeks.

\section{Declaration of conflicting interests}

The authors declared no conflicts of interest with respect to the authorship and/or publication of this article.

\section{Funding}

The authors received no financial support for the research and/or authorship of this article.

\section{REFERENCES}

1. Winthrop KL, Chang E, Yamashita S, Iademarco MF, LoBue PA. Nontuberculous mycobacteria infections and anti-tumor necrosis factor-alpha therapy. Emerg Infect Dis 2009;15:1556-61.

2. Caron J, Michot C, Fabre S, Godreuil S, Guillot $\mathrm{B}$, Dereure O. Aggressive cutaneous infection with Mycobacterium marinum in two patients receiving anti-tumor necrosis factor-alfa agents. J Am Acad Dermatol 2011;65:1060-2.

3. Danko JR, Gilliland WR, Miller RS, Decker CF. Disseminated Mycobacterium marinum infection in a patient with rheumatoid arthritis receiving infliximab therapy. Scand $J$ Infect Dis 2009;41:252-5.

4. Timoney I, Lynch M, Timoney L, Feeney E, Kirby B. Mycobacterium marinum infection contracted from seaweed wrap in a psoriasis patient undergoing treatment with adalimumab. Dermatol Online J 2017;23(8). pii: 13030/qt7zx7m93c.

5. Behr MA, Falkinham JO. Molecular epidemiology of nontuberculous mycobacteria. Future Microbiol 2009;4:1009-20.

6. Ribot E, Poisnel E, De Biasi C, Roudier J, Balandraud N. Atypical Mycobacterium marinum infection (Aquarium granuloma) in a patient on TNF $\alpha$ antagonist therapy for psoriatic arthritis. Joint Bone Spine 2014;81:272-3.

7. Kaneko S, Seishima M, Asano Y, Chinuki Y, Morita E. Mycobacterium marinum infection in a case of psoriasis treated with antitumor necrosis factor $\alpha$ antibody detected by QuantiFERON( $(\circledR)$-TB test. Int $\mathrm{J}$ Dermatol 2014;53:187-9.

8. Demitsu T, Yamada $T$, Umemoto $N$, Narita $T$, Kakurai M, Yoneda K. Cutaneous Mycobacterium marinum infection mimicking felon in a patient with psoriatic arthritis treated with infliximab. J Dermatol 2012;39:970-1.

9. Alkhawaja S, Tammam N, Khalifa N. Mycobacterium marinum infection after infliximab therapy. Iran $\mathrm{J}$ Allergy Asthma Immunol 2010;9:255-7. 